\title{
Interference Avoidance through Dynamic Downlink OFDMA Subchannel Allocation using Intercell Coordination ${ }^{1}$
}

\author{
Mahmudur Rahman and Halim Yanikomeroglu \\ Broadband Communications and Wireless Systems (BCWS) Center \\ Department of Systems and Computer Engineering \\ Carleton University, Ottawa, Ontario K1S 5B6, Canada \\ \{mmrahman,halim\}@sce.carleton.ca.
}

\begin{abstract}
OFDMA subchannel allocation has been wellstudied. However, most of the available literature considers a single cell system without cochannel interference. We present a novel intercell interference avoidance scheme, in which the downlink OFDMA subchannels are allocated through intercell coordination in a multicell environment. The proposed scheme not only aims to achieve maximized network throughput but also focuses on providing improved throughput for cell or sector edge users that are most affected by intercell interference. Enhanced cell edge performance may result in fewer base stations needed to cover a region; this, in turn, may yield substantial savings in deployment cost. The scheme is comprised of two different algorithms; one at the base station level, and the other at a central controller to which a group of neighboring base stations are connected. The performance of the proposed scheme is compared with that of a reference scheme in which coordination is not employed. It is observed from simulation results that the proposed scheme outperforms the reference scheme in terms of network and cell edge throughput.
\end{abstract}

Index Terms- OFDM resource allocation, interference avoidance, radio resource optimization.

\section{INTRODUCTION}

As a promising modulation technique for the next generation wireless systems, such as IEEE 802.16 , 3GPP longterm evolution (LTE), and $4 \mathrm{G}$ cellular (see, for instance, WINNER [1]), orthogonal frequency division multiplexing (OFDM) has attracted significant attention recently. Besides OFDM's inherent ability to combat inter-symbol interference (ISI) resulting from frequency selective fading, orthogonal frequency division multiple access (OFDMA) offers flexibility in radio resource allocation as each subcarrier can be allocated intelligently and modulated adaptively to exploit multiuser and frequency diversities, respectively. As a result, the achievable data rates on the available frequency spectrum can be improved significantly. In this paper, we propose a viable interference avoidance scheme that uses dynamic OFDMA subchannel allocation with aggressive frequency reuse in a multicell environment.

Numerous studies on subchannel allocation in OFDMA systems in a single cell context are available in the literature. In most of these studies, the multiuser diversity gain is explored using various optimization techniques [2-4]. In designing practical networks, however, optimization should be performed in a multicell environment considering one of the major challenges associated with future wireless systems, i.e., intercell interference. To the authors' knowledge, resource optimization in a multicell environment has not yet been investigated well and only a few research works on multicell allocation can be found in the literature [5,6]. In [5], a linear programming (LP) formulation is proposed, where subchannels are partitioned and assigned fixed reuse factors such that user terminals (UTs) at the cell edge can only use subchannels with higher reuse factors. Contrary to the scheme in [5], our proposed scheme does not require any prior resource partitioning or cell planning. While such partitioning of resources reduces resource utilization proactively, our proposed scheme results in a dynamic and efficient reuse factor for each subchannel depending on mutual interference situations and the UTs' data rate requirements. The scheme presented in [6] is interesting from an optimization point, where algorithms residing at the radio network controller (RNC) and base stations (BSs) aim to optimize the total network throughput. However, since the UTs' rate requirements are not considered in the formulation, the scheme is expected to favor UTs that are closer to the BS leading to starvation and unfairness for the cell edge UTs most affected by interference.

In this paper, a chunk is regarded as the minimum granularity of the radio resource allocation unit, which is defined as a collection of consecutive subcarriers over a defined time period. By using intercell coordination, our proposed scheme thrives to maximize throughput on allocated chunks considering the rate requirements of the cell edge UTs. In particular, any optimal or sub-optimal allocation scheme, such as in [6], can exploit multiuser diversity to achieve maximized sector throughput by optimally or sub-optimally assigning best chunks to UTs. However, as the cell edge UTs experience higher path-losses on the desired links and higher interference from nearby cells, these scheduling schemes tend to overlook such disadvantaged UTs. Therefore, interference avoidance is crucial in order to offer minimum required data rate to the cell edge UTs.

The proposed scheme is comprised of two separate algorithms residing at the BS and at a central entity. Based on the interference received by its UTs and their data rate require-

\footnotetext{
${ }^{1}$ This work was supported by the Natural Sciences and Engineering Research Council (NSERC) of Canada under participation in the Wireless World Initiative New Radio (WINNER) project - www.ist-winner.org. Concepts presented in this paper were contributed, in part, to the WINNER deliverable D4.7.2.
} 
-ments, each sector (via its BS) sends a request to the central controller; this request incorporates a tentative list of chunks in the requesting sector. The central controller gathers all such requests and processes to prepare a refined list of chunk restrictions to be applied in all involved sectors in different cells. Once the restriction list is available to a sector, its scheduler allows transmissions only on the eligible chunks.

The performance of the proposed scheme is compared with that of a reference scheme in which intercell coordination is not used and resource allocation is performed in the presence of total interference.

The remainder of this paper is organized as follows. Detailed descriptions of the scheme along with a brief overview of the system under study are presented in Section II. Section III describes simulation models and system parameters. Simulation results are discussed in Section IV. The implementation complexity issues of the proposed scheme are addressed in Section V followed by the conclusions in Section VI.

\section{Descriptions of the Scheme}

The network layout under study is illustrated in Fig. 1. A total of 19 cell sites, each with 3 hexagonal sectors, are considered. Available frequencies are assumed to be reused in each sector as per WINNER baseline system configuration [7]. Sectors are equipped with $120^{\circ}$ directional transmit antennas, while the UTs' receive antennas are considered to be omnidirectional. The antenna gain pattern for the transmit antenna is discussed in the following section.

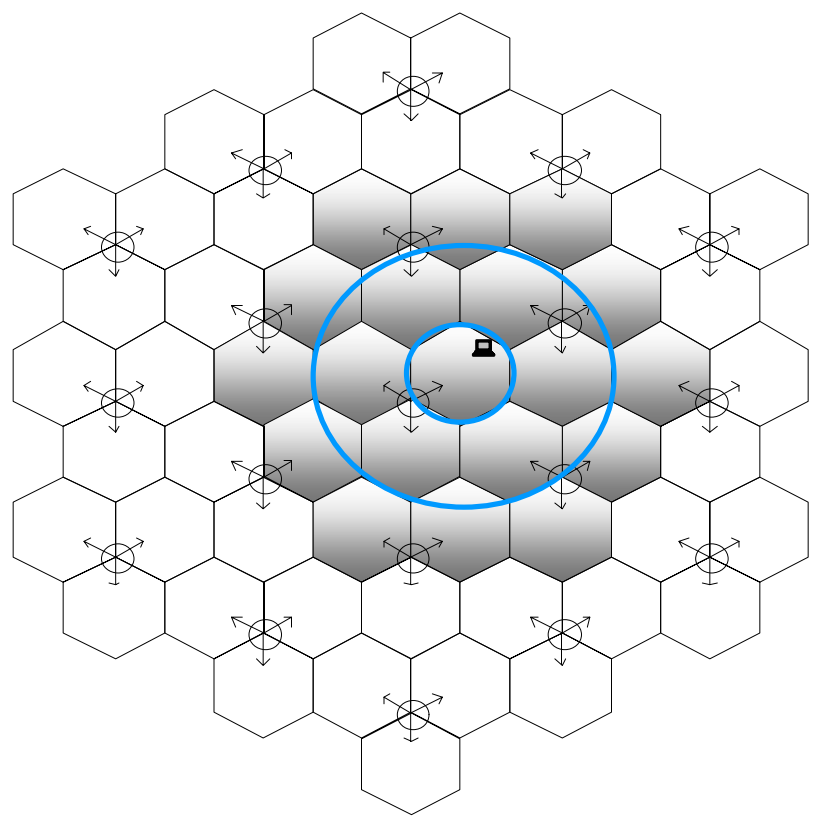

Fig. 1: Network layout under investigation

For the given layout, it is apparent that for a downlink transmission to a UT in any sector (for instance, the sector marked by the smaller circle), its first tier sectors ${ }^{2}$ as enclosed by the wider circle would be the most dominant interferers due to their relative locations and antenna directivities. A cell edge UT, as shown in Fig. 1, experiences higher path-loss and receives significant interference from the sectors of nearby cells. Also, UTs closer to the serving sector may experience severe interference from neighboring sectors of own cell. As a consequence, these UTs are expected to see more poor-quality chunks having low signal-to-interference-plus-noise ratios (SINRs). The optimal or sub-optimal allocation schemes that maximize network throughput may overlook such disadvantaged UTs, which are less attractive to contribute to the total throughput compared to the ones closer to the BS. Therefore, it is very important to avoid interference on these UTs in order to guarantee their minimum required rates.

As mentioned earlier, the proposed scheme is composed of two different algorithms residing at each sector (i.e., BS) and at a central controller. The working principle of the scheme is described by a block diagram in Fig. 2. The algorithm at the sector prepares chunk restriction requests based on the desired and dominant interferer channels as well as the UTs' resource demands derived from the required and achieved rates. In particular, this is a utility maximization problem where the utility measure considers the above mentioned factors as well as the fairness across the sectors. The obvious question is that what happens when a pair of mutually interfering sectors wants to restrict the same chunk from each other. In this case, a central entity is required to resolve the conflict. The algorithm at the central controller solves request conflicts in an optimal manner, where the problem is formulated as a linear constrained problem with binary variables. The details of these algorithms are discussed below.

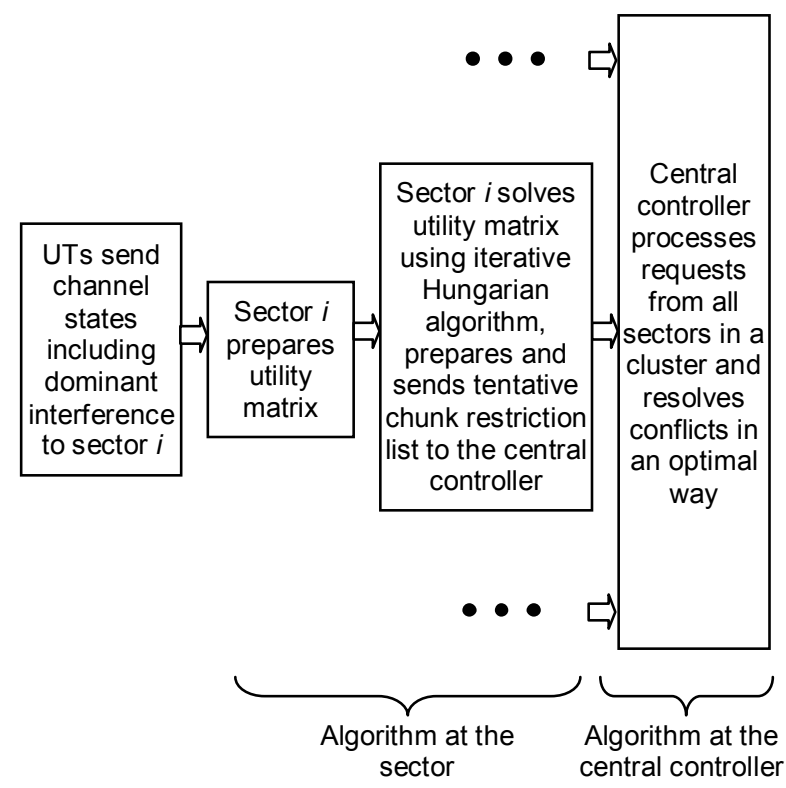

Fig. 2: Block diagram of the scheme

\footnotetext{
2 Dominant interference is received from sector transmit antennas of different cells as well as from its own cell. Intercell coordination implies that these cells coordinate resource usage restrictions at corresponding sector antennas.
} 


\section{A. Sector Algorithm}

Let us denote $j$ and $k$ as the indices of the first and second tier interferers, respectively, for the UTs in sector $i ; m$ and $n$ are the indices for UTs and chunks, respectively. For any chunk $n$, the SINR seen at UT $m$ in sector $i$ can be given by,

$$
\rho_{m, n}^{(i)}=\frac{P_{t} H_{m, n}^{(i),(i)}}{P_{N}+P_{t} \sum_{j=1: 6} H_{m, n}^{(i),(j)}+P_{t} \sum_{k=1: 12} H_{m, n}^{(i),(k)}},
$$

where $P_{t}$ is the transmit power applied on each chunk derived from equal power distribution; that is, $P_{t}=P_{T} / N$, where $P_{T}$ is the total transmit power per sector and $N$ is the number of available chunks. $P_{N}$ is the thermal noise over the chunk bandwidth. $H_{m, n}^{(i),(j)}$ and $H_{m, n}^{(i),(k)}$ are the first and second tier interferer channels on chunk $n$ at UT $m$ in sector $i$. $H$ reflects the large scale path-loss, antenna gain and directivity, fading, and shadowing. The first summation in the denominator has the most dominant effect on the quality of the chunk.

The minimum and maximum rates on chunk $n$, denoted as $r_{m, n(\min )}^{(i)}$ and $r_{m, n(\max )}^{(i)}$, can be achieved from (1) when none and all first tier interferers are restricted, respectively. However, moving from $r_{m, n(\min )}^{(i)}$ to $r_{m, n(\max )}^{(i)}$ implies increasing the penalty to the interferer sectors, as more and more chunks are to be restricted there. The demand factor, $D_{m}^{(i)}$, is defined as the ratio of the target rate to the average rate achieved by UT $m$ in sector $i$. At the sector algorithm, a threshold-based strategy is used to determine which interferers are requested to be restricted as described below.

Based on its demand factor and channel conditions, a UT can restrict two most dominant interferers at most. This limits resulting chunk restrictions in the neighboring sectors and the complexity of the central algorithm. First, a utility matrix $U^{(i)}$ is prepared at each sector. Then, the utility matrix is solved using iterative Hungarian (also known as Kuhn-Munkres) algorithm [8] in order to reserve chunks for its UTs.

In order to build the utility matrix, the following steps are repeated for each UT and chunk.

1) Dominant interferers are sorted in descending order into a dominant interferer set.

2) $r_{m, n(\min )}^{(i)}$ is calculated from the SINR expression in (1) considering the presence of all dominant interferers.

a. If $r_{m, n(\min )}^{(i)} \geq r_{1}^{T H}$, no interferers are requested to be restricted if chunk $n$ is assigned to UT $m$, irrespective of its demand factor. UT $m$ is then either having a strong desired link from serving $\mathrm{BS}$ or is experiencing weak interference from all dominant interferers on chunk $n$. In simulations, we have used $r_{1}^{T H}=3 \mathrm{bps} / \mathrm{Hz}$. b. Else, calculate the new rate $r_{m, n(n e w)}^{(i)}$ with the most dominant interferer being restricted.

i. If $D_{m}^{(i)} \leq 1$ (UT $m$ has been rate-satisfied in the past) and $r_{m, n(\text { new })}^{(i)}-r_{m, n(\min )}^{(i)} \geq r_{2}^{T H}$, UT $m$ will request this dominant interferer to be restricted (here, $r_{2}^{T H}<r_{1}^{T H}$ ). The above conditions imply that for a rate-satisfied UT, interferer restriction is justified only when a considerable gain can be achieved. $r_{2}^{T H}=1 \mathrm{bps} / \mathrm{Hz}$ has been used in simulations.

ii. Else if $D_{m}^{(i)}>1$ and $r_{m, n_{(\text {new })}}^{(i)}-r_{m, n_{(\min )}^{(i)}}^{(i)}>0$, the most dominant interferer will have to be restricted. In some instances, $r_{m, n(n e w)}^{(i)}$ might be zero. In such a case, the second most dominant interferer would also be restricted if doing so provides any achievable rate on chunk $n$ for UT $m$.

After finding the dominant interferer(s) to be restricted on each chunk and each UT, achievable rates $r_{m, n}^{(i)}$ are calculated. Now, the utility of chunk $n$ for UT $m$ can be expressed as:

$$
U_{m, n}^{(i)}=r_{m, n}^{(i)} \times N C_{m, n}^{(i)} \times D_{m, n}^{(i)},
$$

where $N C_{m, n}^{(i)}$ is the number of first tier interferers that UT $m$ allows, yet achieves a rate of $r_{m, n}^{(i)}$. In (2), the parameter $N C_{m, n}^{(i)}$ is used in order for the sector algorithm not to be greedy (i.e., to ensure fairness across sectors). The utility matrix is given by $U^{(i)}=\left[U_{m, n}^{(i)}\right]$. Each entry of $U^{(i)}$ is associated with corresponding interferer(s) to be restricted as well as achieved rate when the chunk is used by the respective UT.

$U^{(i)}$ is solved using the iterative Hungarian algorithm. This is an efficient and low complexity optimal algorithm for one-to-one assignment problem. However, a UT needs multiple chunks in order for it to achieve the required rate, and the iterative Hungarian method becomes a rather good suboptimal solution. Chunks are tentatively allocated (as the central controller might override the restriction requests) in order to reserve chunks for each UT with the following steps.

1) Initialize the rate achieved for each UT: $R_{m}^{(i)}=0$. Initialize the interferer restriction request list to be empty.

2) Apply the Hungarian algorithm to $U^{(i)}$. Update the achieved rates for the assigned chunks, $R_{m}^{(i)}=R_{m}^{(i)}+r_{m, *}^{(i)}$, where $r_{m,{ }^{*}}^{(i)}$ is the rate of optimally assigned chunk to UT $m$. If the utility entry has a corresponding interferer restriction, the restriction list will be updated with the entered interferer. 
3) Delete the columns of the utility matrix corresponding to assigned chunks from the utility matrix. Also, if $R_{m}^{(i)} \geq R_{m}^{r e q}$, delete the corresponding $\operatorname{row}(\mathrm{s})$ of the utility matrix. Here, $R_{m}^{r e q}$ is the required rate for UT $m$. Now, apply the Hungarian algorithm to the updated utility matrix.

4) Repeat steps 2) and 3) until all UTs' rates are satisfied or the resource is exhausted.

5) The restriction request list is now ready to be forwarded to the central controller.

\section{B. Central Controller Algorithm}

The central controller receives requests from a cluster of BSs and resolves conflicting requests in an optimal way. For a particular chunk, Fig. 3 shows an example problem to be solved at the central controller using its algorithm. Here, the green (solid line) and red (dashed line) arrows indicate that interference received at the arrow-originating-sector from the arrowhead-sector is acceptable and unacceptable (requested to be restricted), respectively. For example, for a chunk of interest, sector $B$ can tolerate interference from sector $A$, but the opposite is not true as there is a red (dashed) arrow from sector $A$ toward $B$. In this case, either sector $A$ or $B$ has to be restricted for this chunk.

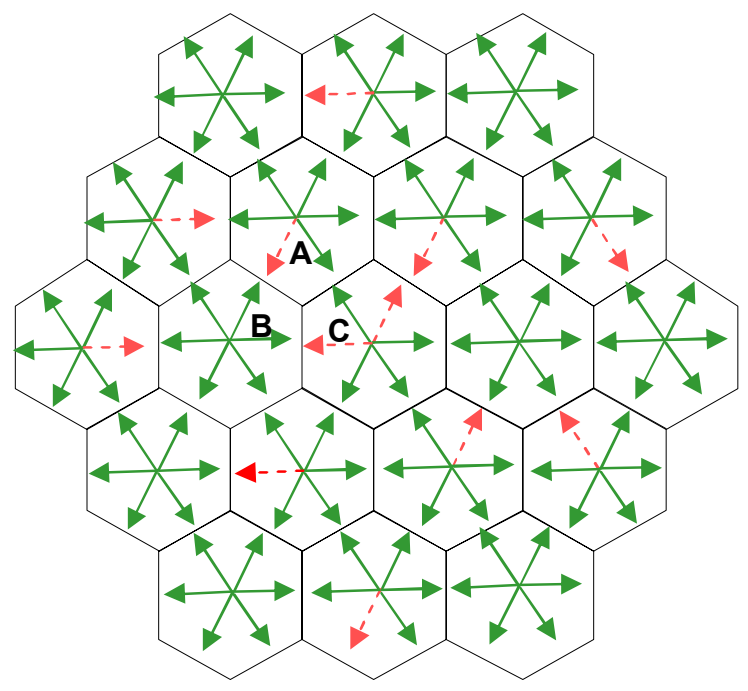

Fig. 3: Chunk restriction request for a particular chunk

The following integer linear optimization problem can be formulated to resolve request conflicts optimally:

$$
\sum_{i, j ; j \neq i}^{\text {Maximize }} U_{m_{i}, n}^{(i)} x_{i, n}+U_{m_{j}, n}^{(j)} x_{j, n} ; \quad \forall n,
$$

where sector $i$ (sector of interest) and sector $j$ (first-tier of dominant interferer) are having conflicting chunk restriction requests for chunk $n$, and $m_{i}$ and $m_{j}$ are candidate UTs for this chunk in sector $i$ and $j$, respectively. subject to

$$
x_{i, n}+x_{j, n} \leq 1 ; \quad \forall n,
$$

where any two sectors $i$ and $j$ have conflicting restriction requests. The variables $x_{i}$ and $x_{j}$ are binary. $x_{i}$ or $x_{j}$ takes a value of 1 if the conflict is resolved in favor of sector $i$ or $j$, otherwise, it is zero.

The central controller resolves request conflicts and sends refined restriction lists to all involved BSs.

The proposed restriction processing can be done from time to time as long as the channel coherence time permits. This period is usually much longer than the scheduling interval and shorter than channel coherence time. Once the chunk restriction list is available at a sector, the scheduler can perform chunk scheduling based on its own criteria. In that sense, the above chunk restriction technique can be considered independent of scheduling. In this study, we assume the iterative Hungarian algorithm for scheduling in both the proposed and the reference schemes. Chunk restriction processing is performed on 10 chunks time interval and scheduling is done at every chunk time duration.

\section{Simulation Models and Parameters}

A total of 19 cell sites (i.e., 57 hexagonal sectors) are considered, as shown in Fig. 1. The inter-site distance is $1 \mathrm{~km}$. UTs are randomly distributed [9] in the centre 19 shaded sectors within a minimum and maximum radius in each sector. While the sector algorithm is executed in these 19 sectors, other sectors remain as interference contributors only. Performance statistics are collected from 7 centre sectors (inside the wider circle).

$45 \mathrm{MHz}$ spectrum in the $3.95 \mathrm{GHz}$ frequency band is available to each sector giving a total of 1152 subcarriers each of which has a bandwidth of $39.0625 \mathrm{kHz}$. A chunk (subchannel) consists of 8 consecutive subcarriers. It is a timefrequency resource unit occupying $0.3456 \mathrm{~ms}$ and $312.5 \mathrm{KHz}$, which translates into $8 \times 12$ OFDM symbols [7].

All UTs are assumed to fall in the same service class having a downlink rate requirement of 5.6 Mbps (i.e., 1920 bits per chunk time duration). For the simplicity of simulations, a full queue model has been considered for the downlink traffic.

Rayleigh channel samples, correlated in time and frequency, are generated from the power delay profile for WINNER wide area scenario [9]. UT mobility is assumed to be $20 \mathrm{~km} / \mathrm{hr}$. The following path-loss $(L)$ model assuming pathloss exponent of 3.5 has been used as defined in [9],

$$
L=38.4+35.0 \log _{10}(d)[\mathrm{dB}],
$$

where $d$ is distance in meters between transmitter and receiver.

While UTs are equipped with omnidirectional receive antennas, the gain pattern for $120^{\circ}$ directional sector transmit antennas is considered to be as follows [7]:

$$
A(\theta)=-\min \left[12\left(\frac{\theta}{\theta_{3 d B}}\right)^{2}, 20\right][\mathrm{dB}],
$$


where the value of $\theta_{3 d B}$ is $70^{\circ}$ and $\theta$ varies from $-180^{\circ}$ to $180^{\circ}$. We have used a single-in-single-out (SISO) antenna configuration.

A noise figure of $7 \mathrm{~dB}$ is used to calculate average thermal noise power. Independent lognormal random variables with a standard deviation of $8 \mathrm{~dB}$ are considered for shadowing. Transmit power is assumed to be 39.81 Watts per sector and chunks are assigned fixed equal powers. Retransmission for erroneous data is not considered.

Adaptive modulation with a block low-density paritycheck (B-LDPC) code is used. Thresholds for transmission schemes are determined assuming a block length of 2304 bits and $10 \%$ block error rate (BLER) as shown in Table I [7]. The corresponding water-fall curves were produced from link level simulations. Here, a chunk using QPSK with a coding rate of $1 / 2$ can carry 96 information bits.

TABLE I

LOOKUP TABLE FOR AMC MODES

B-LDPC WITH BLOCK LENGTH OF 2304 BITS AND BLER OF 10\%

\begin{tabular}{c|c|c}
\hline SINR Range $(\mathrm{dB})$ & AMC Mode & $\begin{array}{c}\text { Efficiency, } \eta \\
(\mathrm{Bits} / \mathrm{Sec} / \mathrm{Hz})\end{array}$ \\
\hline \hline$-1.76 \leq \gamma<0.14$ & BPSK rate 1/2 & 0.5 \\
$0.14 \leq \gamma<1.15$ & BPSK rate 2/3 & 0.67 \\
$1.15 \leq \gamma<3.14$ & QPSK rate 1/2 & 1.0 \\
$3.14 \leq \gamma<4.15$ & QPSK rate 2/3 & 1.33 \\
$4.15 \leq \gamma<6.55$ & QPSK rate 3/4 & 1.5 \\
$6.55 \leq \gamma<9.01$ & 16-QAM rate 1/2 & 2.0 \\
$9.01 \leq \gamma<10.22$ & 16-QAM rate 2/3 & 2.67 \\
$10.22 \leq \gamma<14.01$ & 16-QAM rate 3/4 & 3.0 \\
$14.01 \leq \gamma<15.33$ & 64-QAM rate 2/3 & 4.0 \\
$\gamma \geq 15.33$ & 64-QAM rate 3/4 & 4.5 \\
\hline
\end{tabular}

In order to solve the binary integer optimization problem at the central controller, YALMIP [10] and LPSOLVE (an integer linear programming (ILP) solver) [11] have been used along with MATLAB.

\section{Simulation Results}

Performance results have been observed for a user density of 10 UTs per sector. Performance of the proposed scheme is compared with that of a reference scheme where intercell coordination is not used. The reference scheme copes with intercell interference and maximizes throughput considering UTs' rates in the presence of all interferers. While both schemes achieve multiuser diversity, the gain in the proposed scheme is solely due to interference avoidance.

SINRs are predicted on each chunk from the pilot signals. As the reference scheme does not use intercell coordination, the predicted SINRs are determined conservatively based on the assumption that all other sectors are using any particular chunk concurrently. The cumulative distribution functions of the SINRs seen on scheduled chunks for both schemes are shown in Fig. 4. The proposed scheme shows an improvement of $2.5 \mathrm{~dB}$ in the $50^{\text {th }}$ percentile of chunk SINRs. However, this SINR improvement does not have a complete meaning without taking throughput into account.

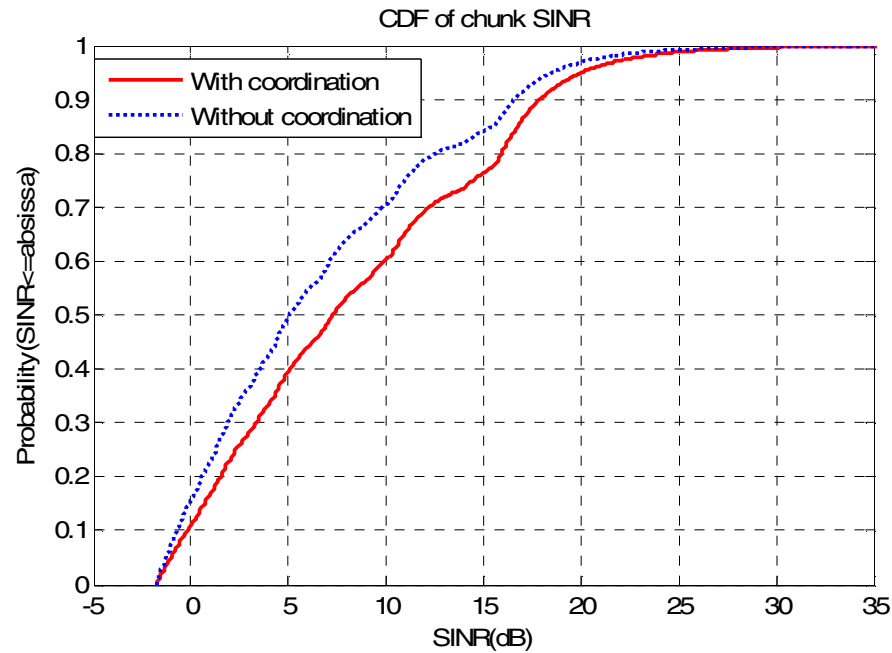

Fig. 4: CDF of chunk SINRs on scheduled chunks

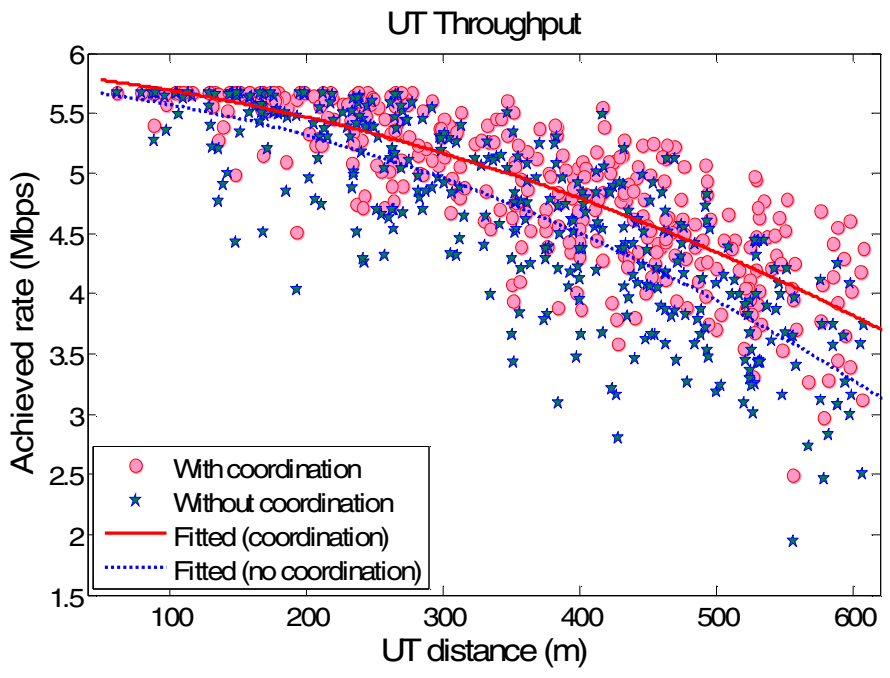

Fig. 5: Scatter plots and fitted curves of UT throughput

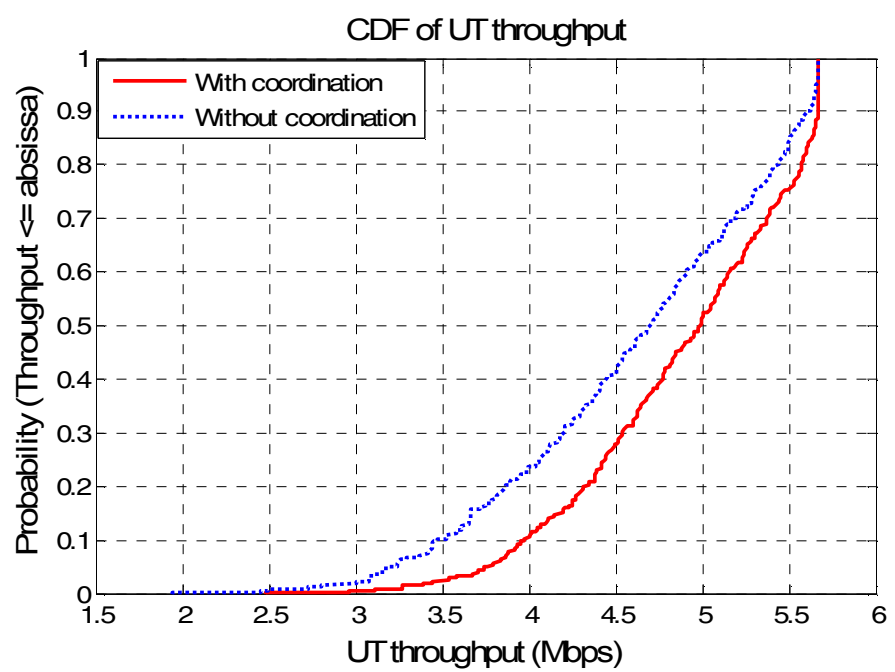

Fig. 6: CDF of UT throughput 
Fig. 5 shows achieved UT throughput for both schemes in scatter plots along with quadratic-fitted curves. It is observed from the figure that up to $20 \%$ throughput gain can be achieved at the cell edge UTs in the proposed scheme with coordination. This improvement is crucial from the user satisfaction point. For example, as shown in Fig. 6, around $90 \%$ of the UTs enjoy a data rate of $4 \mathrm{Mbps}$ or more with the proposed avoidance scheme, while only $77 \%$ do when coordination is not used.

\section{IMPLEMENT ATION COMPLEXITY ISSUES}

The computational complexity of the algorithms and the signaling overhead complexity are two different kinds of complexities associated with the proposed scheme.

The overall complexity of the sector algorithm is dominated by the complexity of the Hungarian assignment algorithm. This combinatorial optimization algorithm solves the assignment problem in polynomial time with worst-case complexity of $O\left(N^{3}\right)$, where $N$ is the number of chunks. For each chunk, the complexity of the algorithm at the central controller depends on the number of sectors that have conflicting restriction requests. This determines the number of variables and constraints involved in the binary integer problem. For example, if there are 3 pair-wise conflicting requests for a particular chunk, then the number of binary variables to solve is 6 and the number of constraints is 9 . Moreover, there are efficient ways to solve such integer problems. For instance, LPSOLVE first finds a relaxed linear feasible solution efficiently and then applies branch and bound iterations for the optimal solution in order to reduce the overall complexity.

The amount of signaling overhead in a certain time interval is related to the frequency of the channel reporting and resource allocation operations, which essentially depends on the mobility of the UTs and hence the resulting channel coherence time. For a UT moving at $20 \mathrm{~km} / \mathrm{hr}$, the channel coherence time is around $6 \mathrm{~ms}$. In this case, channel reporting and resource allocations have to be performed within every 18 chunks time duration. However, for a speed of $70 \mathrm{~km} / \mathrm{hr}$, the time interval of this channel reporting would be around 5 chunks time duration. Therefore, a higher signaling overhead would have to be supported for higher mobility UTs. The signaling overhead bottleneck would be over-the-air channel reporting from UTs to BS; the signaling between BS to the central controller can be performed using high data rate backbone connections such as fiber links and thus it is less of an issue.

Furthermore, in order to reduce both the computational and overhead complexities further, the proposed scheme could potentially be applied to the cell edge UTs only, for which it is needed most. Simulations with this reduced number of UTs remain for future study.

\section{Conclusions}

A novel viable interference avoidance scheme using downlink multicell chunk allocation with dynamic intercell coordination is presented in this paper. According to simulation results, the scheme outperforms the reference scheme not only in terms of higher total throughput, but also in achieving better satisfaction and fairness for the cell/sector edge UTs. It should be noted that the reference scheme exploits similar multiuser diversity as the proposed scheme, since both use the Hungarian algorithm for scheduling. The reference scheme maximizes throughput considering the mutual interference and UTs' data rate requirements. Therefore, the observed performance gain in the proposed scheme is solely due to interference avoidance. Enhanced cell edge throughput in the proposed scheme can potentially allow a smaller number of BSs to cover a region yielding substantial savings in the deployment cost.

In the sector algorithm, a simple utility function has been used, where the considered utility is proportional to the achieved rate on the chunk of interest, UT resource demand at the allocation instant, and the number of allowed concurrent transmissions in the first tier sectors. However, it may be possible to devise a better utility function that may consider other critical factors for better network performance. Also, in order to reduce complexities, scheme may be modified to allow only UTs with poor rate to restrict chunks in the dominant interferers. These issues remain under investigation at the time of writing this paper.

\section{ACKNOWLEDGEMENTS}

The authors would like to express their gratitude to Dr. Abdulkareem B. Adinoyi for his review and useful comments on the manuscript.

\section{REFERENCES}

[1] Wireless World Initiative New Radio, www.ist-winner.org.

[2] C.Y. Wong, R.S. Cheng, K.B. Letaief, and R.D. Murch, "Multiuser OFDM with adaptive subcarrier, bit, and power allocation," IEEE Journal on Selected Areas in Communications, vol. 17, no. 10, pp. 17471757, October 1999.

[3] W. Rhee and J.M. Cioffi, "Increase in capacity of multiuser OFDM system using dynamic subchannel allocation," in Proc. IEEE Vehicular Technology Conf., vol. 2, pp. 1085-1089, May 2000.

[4] D. Niyato and E. Hossain, "Adaptive fair subcarrier/rate allocation in multirate OFDMA networks: radio link level queuing performance analysis," IEEE Transactions on Vehicular Technology, vol. 55, no. 6, pp. 1897-1907, November 2006.

[5] H. Kim, Y. Han, and J. Koo, "Optimal subchannel scheme in multicell OFDMA systems," in Proc. IEEE Vehicular Technology Conf., vol. 3, pp. 1821-1825, May 2004.

[6] G. Li and H. Liu, "Downlink dynamic resource allocation for multi-cell OFDMA systems," in Proc. IEEE Vehicular Technology Conf., vol. 3, pp. 1698-1702, October 2003.

[7] Test Scenarios and Calibration Cases Issue 2, WINNER Deliverable D6.13.7, December 2006, https://www.ist-winner.org.

[8] H.W. Kuhn, "The Hungarian method for the assignment problem," Naval Research Logistic Quarterly, vol. 2, no. 1, pp. 83-97, 1955.

[9] Final report on link level and system level channel models, WINNER Deliverable D5.4, November 2005, https://www.ist-winner.org.

[10] J. Löfberg, "YALMIP: a toolbox for modeling and optimization in MATLAB," In Proceedings of the CACSD Conference, Taipei, Taiwan, 2004.

[11] M. Berkelaar, K. Eikland, and P. Notebaert, lp_solve version 5.5, http://tech.groups.yahoo.com/group/lp_solve. 